\section{PRODUCTION OF CONTRAST IN THE MICROSCOPE IMAGE BY MEANS OF OPAQUE DIAPHRAGMS}

\author{
By DR. A. F. HALLIMOND \\ Geological Survey and Museum
}

\begin{abstract}
$\mathrm{P}$ HASE-CONTRAST' methods have recently been widely used to improve the visibility of small transparent objects. The illumination is restricted to a narrow cone by means of an annular diaphragm placed under the condenser, and the direct beam passes through a conjugate annular 'phase-plate' near the upper principal focus of the objective. A theoretical explanation of the effect was given in the original paper by Zernike. It appears to me, however, that Zernike's explanation is insufficient through the omission of an important physical factor, which seems to have been only partly recognized in the later paper by Kohler and Loos. "The details of such an object (transparent) are distinguished only by unequal thickness or refractive index, i.e., they
\end{abstract}

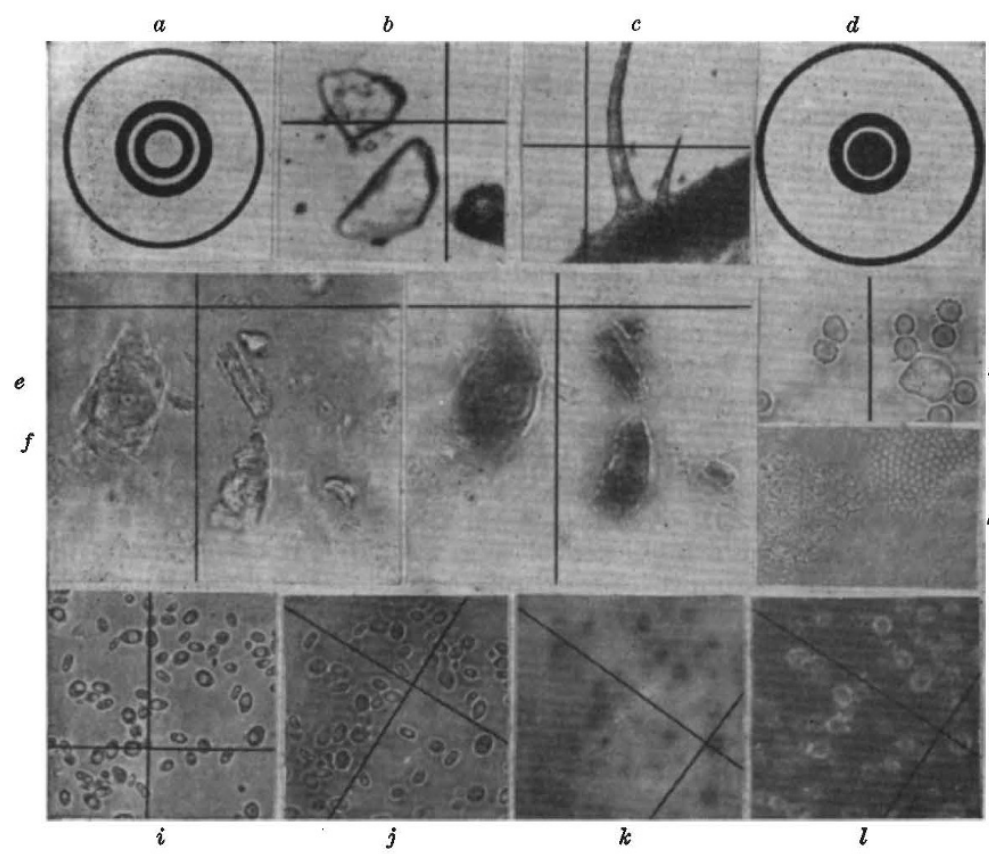

a. Contrast diaphragm at the back focal plane of the $25 \mathrm{~mm}$. objective used for $b, c$. $b$. Objective $25 \mathrm{~mm}$. with contrast diaphragm $a$ above the objective. Quartz grain in oil of index a me 1.55 refractive index difference about 0.01 . The steep edges are dark and the rest shaded according to the inclination of the conchoidal surface. $\times 36$.

$c$. As in $b$. A leaf-hair in water. There is little injury to the defnition. $\times 36$ d. Contrast diaphragm at back of $4 \mathrm{~mm}$. objective used for $f, g, h, j, k, l$. $e .4 \mathrm{~mm}$. 85 N.A., no diaphragm above objective, the condenser giving ordinary conical illumination. Scales and rouleaux of kaolinte in balsam, refractive index difference about $0 \cdot 020$. Detail is clear with marked Becke lines, but there is littie contrast with the background. $\times 300$

f. $4 \mathrm{~mm}, 0.65 \mathrm{~N}$.A., contrast diaphragm above the objective as $d$, etc. Detail is still clear, but there is now a marked contrast with the background. $\times 300$.

$g$. As in $f$. Red and white blood corpuscles. $\times 300$.

$h$. As in $f$. Diatomite in liquid of refractive index $1 \cdot 39 . \quad \times 300$

i. $4 \mathrm{~mm}$. 0.85 N.A., no diaphragm above the objective, ordinary conical illumination as in $e$. Yeast in water. Some of the cells in these photos have moved during the exposures. $\times 300$

j. 4 mm. 0.65 N.A., contrast diaphragm above the objective as in $f$, etc. Yeast is rather strongly refractive and there is little addition to the detall already visible in $i$. No injury to definition.

$k$. As in $j$. The outer zone of the contrast diaphragm $d$ has been covered so that only the direct beam through the annulus is being used. There is considerable loss of definition, the focus in this case being very deep.

$l$. As in $j$. The annulus has been covered so that only the outer zone of the contrast diaphragm $d$ is being used. This gives in effect a 'dark ground' illumination, and the image chiefly consists of bright lines due to the steep edges of the cells. (The accompanying photographs by an arrangement of opaque stops, without the thod from that involving the use of the in plate.

In phase-contrast, if a given 'point' in the object phase from that which may still traverse the phaseplate, and the image is consequently weakened by interference. By adjusting the opacity of the phaseount of light in the two parts of the beam can be matched so as to produce maximum interference. Such an explanation would seem to account for many of the results obtained by phase-contrast, though the full range of these effects has scarcely yet been investigated. The interference method is very efficient in extinguishing the light, but it is open to the objection that when the phase-plate has been adjusted to give maximum contrast for a certain small angular deviation, the more deviated light from other points in the object will mainly miss the phase-plate and will not be extinguished. In such cases an opaque diaphragm may give a wider range of extinction.

Stop-contrast is obtained by using a narrow annular opening below the condenser (in the present case a lens of about $1 \frac{1}{2}$ in. focal length), with a conjugate annular opening near the upper principal focus of the objective. Light deviated by the object is eliminated by surrounding the open annulus at the objective with an opaque diaphragm, which in its simplest form might cover the rest of the objective. Such an arrangement is, however, inadmissible since it reduces the aperture and damages the resolving power (photo. $k$ ). The opaque part of the stop must therefore be usually limited to a certain angular distance immediately on each side of the annulus $(a)$. It is sometimes convenient to darken the whole of the relatively small central area $(d)$. With a complete stop, practically all the deviated light contributing to the Becke and Schroeder effects would be eliminated. With the dark annuli some extremely deviated rays will still get through the open 
outer zone, so that steep edges and objects of high refractive index difference may still show a modified Becke line ; but the desired object may be practically free from it, dark contrast being produced instead.

The following incomplete statement indicates the angular values concerned. The maximum deviation that can be produced by a cylindrical object is that for a ray entering and emerging at the grazing angle and will equal $2(90-\theta)^{\circ}$, where $\theta$ is the critical angle. For refractive indices $1 \cdot 33$ (water) and, say, $1 \cdot 35$ (object), $2(90-\theta)$ is $20^{\circ}$ in the liquid. If the opaque $p$ art of the diaphragm at the back of the objective intercepts rays that occupy this angle, such an object will appear dark except at points near the centre. For low-power objectives with low numerical aperture, the angles that can be covered are much narrower than this, but the method will be sensitive to small differences in refractive index. Higher powers, on the other hand, with numerical aperture about $\mathbf{l} \cdot 0$, will accommodate annuli covering a wider angle that may approach $20^{\circ}$, and they can be made effective for objects of wider deviation.

The accompanying photographs illustrate the results obtainable with low- and medium-power objectives (Cooke, $25 \mathrm{~mm}$., $0.15 \mathrm{~N} . \mathrm{A}$. and $4 \mathrm{~mm}$. N.A. 0 65), by means of narrow conical illumination with opaque stops partly covering the back of the objective as shown in $a$ and $d$. In these cases the depth of focus is very small and only parts of the object can be in focus, the tendency being to obtain an 'optical section'. Phase-contrast photographs of kaolin were reproduced in Nature, 158, 621 (1946).

The comparison photographs $e$ and $i$ were taken with narrow conical illumination. This is probably the most satisfactory form of ordinary illumination for objects of small refractive index difference, since it gives a moderate Becke effect without undue loss of light, but the image is mainly due to this effect.

Photographs $c, f, g, h, j$ indicate that the contrast diaphragm causes no great injury to the resolution of details in the object. $k$ and $l$ were taken through the annulus and outer zone of the objective, used separately. Neither would supply the detail observed in $j$, although the only difference is that in $j$ both areas are open simultaneously. The improvement is presumably due to interference in accordance with Abbe theory.

In ordinary narrow-aperture illumination, a small object in liquid of lower refractive index appears light when the tube is raised, almost invisible when in the focal plane, and dark when the tube is lowered. For a simple isolated object the latter effect resembles that given by contrast methods, but is only produced when the object is somewhat out of focus. When the object is in the focal plane, the use of a contrast diaphragm produces a similar darkening, and the details are in focus. For a complex rugose (or finely granular) object such as the kaolin scales in $e$, the Becke lines overlap in the interior of the object areas, producing a confused mottling which in fact only an experienced observer would interpret as due to a solid object. It is in such cases that the use of contrast methods seems most likely to be advantageous.

The annulus in $d$ was purposely made narrow to test the effect on resolution. It is not suggested that this would be necessary in all cases.

This communication has been published by permission of the Director, Geological Survey and Museum.

\section{INDUSTRY AND RESEARCH IN THE SHEFFIELD AREA}

$T$ HE Federation of British Industries has been holding in recent months a series of conferences on that vital question of the present time, the dependence of progressive industry on research. One of these regional conferences was held in Sheffield on June 5 to discuss the bearing of research on industry in the case of the smaller manufacturing concerns. The problems were considered from two aspects: the first, at a morning session, presided over by Sir Walter Benton Jones, dealt with the part research could play in promoting industrial efficiency, exports and a higher standard of living, with special reference to the industrial problems of the Sheffield area. Sir Walter, in opening the meeting, directed attention to the valuable conclusions of previous conferences, and stated that industry values highly the growth of special research. The afternoon session, Lord Dudley Gordon being in the chair, was devoted to the case of the smaller manufacturing concern which could not afford the cost of a research department, and consideration was given to the research organisations and facilities which could serve it.

The subject of "Research and National Recovery" was discussed by Sir William Larke in a paper full of wise advice of an encouraging and stimulating character. He pointed out that our industrial production needs to be increased above that of 1938 by at least 25 per cent under conditions of a shortage of raw materials and man-power. These requirements point to the need for technical advances, at least as great as those which were achieved during the War. He regards shortage of coal as the principal factor preventing our economic recovery. This difficulty demands still more attention to efficient use, by increased supervision, by continuing to demand of the suppliers fuel of the appropriate quality, and by the provision of more efficient fuel-using plant. Further, our general production efficiency must be at a higher level than that of our competitors. The support of research and closer collaboration of all elements concerned with industry and research are necessary. A survey made by the Federation of British Industries of research activities in British industry has shown a total of some 40,000 staff, of whom 10,000 are graduates of a university or equivalent standard. The total expenditure by industry on research, Sir William said, has increased fourfold in the last ten years, and is now in excess of $£ 25,000,000$ per annum, or at least 0.5 per cent of the total value of our annual industrial production. This situation and the potentialities of rapid application of the results of research and discovery to the improvement of industrial efficiency should provide the means of strengthening our competitive power in world markets. The achievements in the face of the overwhelming difficulties of 1940 onwards give hopes of inspiration and encouragement for the future.

Dr. G. P. Jones, discussing the lessons of history in a paper entitled "The Historical Growth and Economic Development of Sheffield Industries", had perforce to limit his scope to steel-making and cutlery. He showed how much we are dependent on the competence of our forefathers, and in what degree an easy livelihood and survival of two terrible wars are owed to their efforts. The historical aspects of the question were also dealt with by Lord Dudley Gordon in reference to valuable contributions 